\title{
Research on channel estimation algorithm based on compressive sensing
}

\author{
Chang Juan $^{\mathrm{a}}$, Shi Yuhe ${ }^{\mathrm{b}}$ and zhao Hongyan ${ }^{\mathrm{c}}$ \\ Science institute, Air Force Engineering University, Xian, China \\ a34521193@qq.com, byuheshigo@163.com, czyr392@sina.com
}

Keywords:OFDM; underwater acoustic communication; channel estimation compresive sensing.

\begin{abstract}
For OFDM underwater acoustic communication system, OMP and CoSaMP channel estimation algorithm based on the sparsity of underwater acoustic channel are studied and comparised. Both of those algorithms have high estimation accuracy. However, the CoSaMP algorithm has the global optimality of channel reconstruction, and better ensures the stability of channel estimation. Through the simulation analysis, not only the efficiency and accuracy of those two estimation algorithms are verified, but also the optimum number of pilots used in CoSaMP algorithm is found. which at the same time ensuring that the estimation accuracy can effectively reduce the throughput of the system, improve the utilization of spectrum. The CoSaMP algorithm adopting the optimum number of pilots could increase the throughput of the system, and improve the spectrum utilization effectively, when ensuring the estimation accuracy.
\end{abstract}

\section{Introduction}

OFDM is a modulation technique with high spectral efficiency, that has become the new communication technology. Underwater acoustic(UWA) channel with the characteristics of complexity, polytrope, strong severe multipath interferences and limited band, is a very complex channel. To ensure OFDM communication system performance not be affected by the multipath and the fading effect of underwater acoustic channel, the system often uses channel estimation to track the channel response. Compressive Sensing $(\mathrm{CS})^{[1]}$ is a new signal sampling method, that breaks the traditional Nyquist sampling method and could sample the signal with far below Nyquist sampling rate, and could recover the original signal accurately. But only if the sampled signal is sparse in a transform domain. UWA channel is exactly sparse multipath. Thus CS is used in UWA channel estimation, which could improve the channel estimation's precision and validity.

In this paper, the application of OMP and CoSaMP channel estimation algorithm in OFDM UWA communication system based on CS are studied. And the performance of those two algorithms are comparised through the simulation.

\section{Compressive Sensing}

The sampling of CS is firstly to collect the signal's non-adaptive linear projectives(the measurements), then to reconstruct the original signal from measurements according to the reconstruction algorithm. CS is a new signal processing method merging compression and collection. The CS theory includes three aspects: signal sparse representation, design of measurement matrix, reconstruction algorithm [2-4]. The superiority of CS is that the amount of signal's meaturing datas is far lower than traditional sampling method, which reduces the costs of signal's collection and high resolution transmission.

The length of a one-dimensional discrete signal $\mathrm{X}$ is $\mathrm{N}$, that could be expressed as the combination of $\mathrm{N} \times 1$-dimensional basis vectors $\left\{\psi_{i}\right\}_{i=1}^{N}$.

Where those vectors are assumed to be orthonormal bases. The set of basis vectors $\left\{\psi_{i}\right\}_{i=1}^{N}$ as column vectors composes a $\mathrm{N} \times \mathrm{N}$-dimensional matrix $\Psi$, and an arbitrary $X$ could be expressed as 
$X=\Psi \Theta$, where $\Theta$ is $\mathrm{N} \times 1$-dimensional column vectors composed by the projection coefficient $\theta_{i}$.

If $\Theta$ is sparse, an observation basis $\Phi: \mathrm{M} \times \mathrm{N}(\mathrm{M}<<\mathrm{N})$ uncorrelated with transform basis, is used to linearly transform the coefficient basis $\Theta, Y=\Phi \Theta=\Phi \Psi T X$, to obtain the observation set $Y$ : $M \times 1$. Then the original signal $X$ could be reconstructed from the observation set accurately, using optimization method.

\section{UWA Channel Model Based on $\mathrm{CS}^{[5-7]}$}

The transmitted signal in passband can be written as:

$$
\mathrm{z}(t)=\operatorname{Re}\left\{\left[\sum_{k \in S} d[k] e^{j 2 \pi k \Delta t t}\right] e^{j 2 \pi f_{c} t}\right\}, t \in\left[0, T+T_{g}\right]
$$

Where $\mathrm{T}$ and Tg are denoted the OFDM duration and the guard interval respectively, $\Delta \mathrm{f}$ is the subcarrier spacing, fc is carrier frequency, $d[\mathrm{k}]$ is the information symbol to be transmitted on the kth subcarrier, and $\mathrm{S}$ is the set of subcarriers.

UWA Channel has the characteristic of frequency selectivity and time-varying, especially sparsity. Therefore the impulse response of the multipath underwater channel is written as:

$$
\begin{aligned}
& h(t, \tau)=\sum_{p} A_{p}(t) \delta\left(\tau-\tau_{p}(t)\right) \\
& \tau_{p}(t) \approx \tau_{p}-a t
\end{aligned}
$$

Where $A_{p}(t)$ is the p path amplitude, $\tau_{p}(t)$ is the p path delay.

In this model, we treat the channel as having a common Doppler shift on all propagation paths.

Then the recieved signal in passband is:

$$
Y=H Z+V=Z^{T} \otimes I_{K} \text { vec }(H)+V
$$

Where $Z^{T} \otimes I_{K}$ can be considered as $\Phi$ in CS and vec $(H)$ can be considered as $\Psi T X$, and then the UWA Channel estimation can be realized by CS theory.

\section{Channel estimation based on compressive sensing ${ }^{[8-9]}$ \\ OMP algorithm}

The basic idea of the OMP channel estimation algorithm is: firstly the column vector matching with the observation vector $y$ is selected from the observation matrix in every iterative process time to approximate sparsely the underwater acoustic channel h; secondly The column vector selected is orthogonalized and projected to obtain the signal's residual components, samely the column vector removed; thirdly the residual components are decomposited in the same way, after a certain number of iterations. This algorithm can directly find the location and size of the nonzero elements in unknown sparse channel h. The specific steps are as follows:

1. Initialize: the number of iterations $\mathrm{j}=0$, the residual component $\mathrm{r} 0=\mathrm{y}$, index set $\mathrm{S} 0=\varnothing$;

2. The j iteration

a. Determine the index $\mathrm{Sj}$ making

$$
\left.\left|<y_{j-1}, z_{s_{j}}\right\rangle\left|=\max _{S \in\{1, \cdots, N\} \backslash S_{j-1}}\right|<y_{j-1}, z_{s}\right\rangle \mid
$$

where $z_{s}$ represents the $S$ column of the transmission matrix. Because the orthogonality of the vector's $z_{s}$ can't be guaranteed, the orthogonal process is carried in advance.

b. Update the index set $S_{j}=S_{j-1} \cup\left\{s_{j}\right\}$, and obtain new estimated value $\widehat{h}_{j}$, this new estimated value outside the index set $S_{j}$ are zero. the estimated value $\widehat{h}_{j \mid S_{j}}$ in the index set $S_{j}$ value is:

$$
\widehat{h}_{j \mid s_{j}}=\arg \min \left\{\left\|y-Z_{s_{j}} h\right\|^{2}\right\}=Z_{s_{j}}{ }^{-1} y
$$


Where $Z_{s_{j}}$ is the $M \times j$ matrix, which contains the $S_{j}$ column in matrix $Z$.

c. Update the residual components $y_{j}=\mathrm{Z} \hat{h}_{j}=\mathrm{Z}_{s_{j}} \hat{h}_{j \mid s_{j}}, \quad r_{j}=y-y_{j}$

The above iterative steps are repeated until the iteration times reach the sparsity $K$, or the iterative error meets the requirements of stopping the iteration.

After $j$ iterations, the vector $\hat{h}$ whose sparsity is $K$ is obtained, which is the system's channel estimated value.

\section{CoSaMP algorithm[10]}

OMP algorithm is the pursuit of local optimality, simple calculation and fast calculation speed. But the relative residual of the atomic selection mechanism can not be guaranteed is the optimality in every iterative process. Ultimately, the global optimality of channel reconstruction can not be guaranteed. But CoSaMP algorithm cites the idea back when choicing the atomic, at the same time as ensurance of the reconstruction accuracy and low computational complexity, and guarantees the global optimality of channel reconstruction effectively. The algorithm has good properties of robustness and stability.

1. Initialize: the number of iterations $\mathrm{j}=0$, the residual component $\mathrm{r} 0=\mathrm{y}$, index set $\mathrm{S} 0=\varnothing$;

2. The $\mathrm{j}$ iteration the residual component

a. Calculate $\left|<y_{j-1}, z_{s_{j}}\right\rangle \mid$ respectively, where $s \in\{1, \cdots, N\}, z_{s}$ represents the $s$ column of the transmission matrix. And the $2 K$ largest tap positions are founded out to constitute the set $S_{2 K}$.

b. Update the index set $S_{j}=S_{j-1} \cup\left\{s_{2 K}\right\}$, and obtain new estimated value $\widehat{h}_{j \mid S_{j}}=Z_{s_{j}}{ }^{+} y$.

Where $Z_{s}^{+}$is the pseudo inverse matrix of $Z_{s}$, and $Z_{s}^{+}=\left(Z_{s}^{*} Z_{s}\right)^{-1} \cdot Z_{s_{j}}$ is the $M \times j$ matrix, which contains the column whose index is $S_{j}$ in matrix $Z$. The new estimated values outside the index set $S_{j}$ are all zero.

c. From the channel estimated value obtained by that formula, retain $K$ elements as the main tap coefficients, and other taps are set to zero. Therefore the channel estimation result of the $j$ times of iteration is obtained.

d. Update the residual components $y_{j}=Z \hat{h}_{j}=Z_{s_{j}} \hat{h}_{j \mid s_{j}}, \quad r_{j}=y-y_{j}$

The above iterative steps are repeated until the iteration times reach the sparsity $\mathrm{K}$, or the iterative error meets the requirements of stopping the iteration.

Judge whether the iterative error meets the conditions of stopping the iteration. If the iterative error meets the conditions, stop the iteration and output the underwater acoustic channel impulse response estimated value. Otherwise, repeat the above steps, until the iterative error meets the conditions.

\section{Algorithm simulation and analysis}

Figure 1 is the flow chart of the UWA communication system with OFDM.. In this system, we make the Symbol timing synchronization for estimation accuratoin, before Doppler shift estimation. Furthermore, in order to remove the multi-path propagation's effect on the UWA communication system drastically, the channel equalization is adopted.

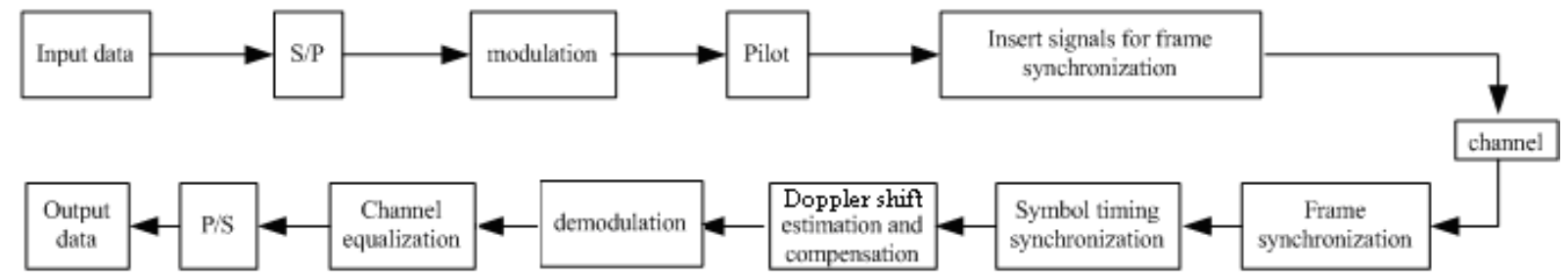

Figure1. The flow chart of the UWA communication system with OFDM. 


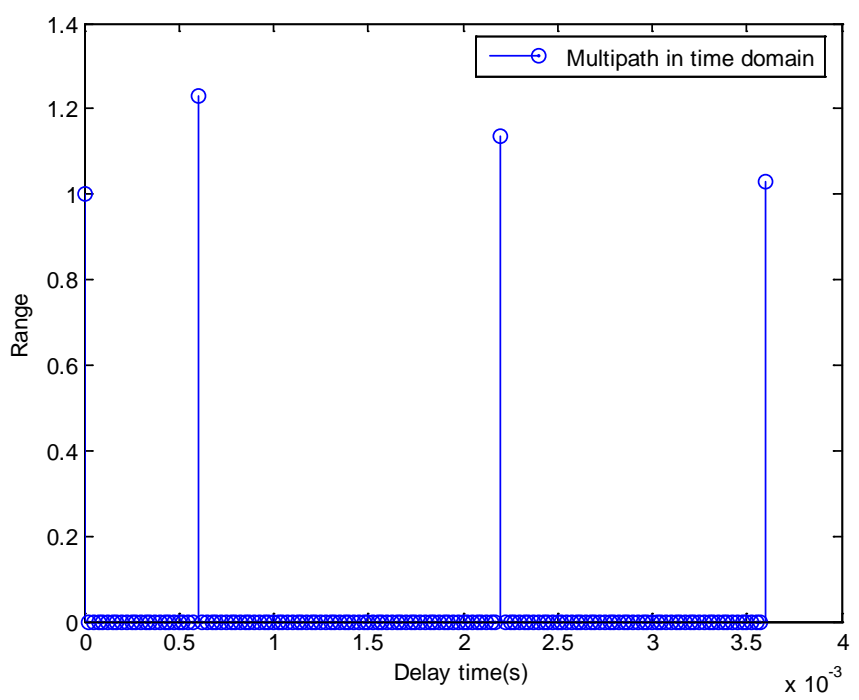

Figure 2. The multi-path channel

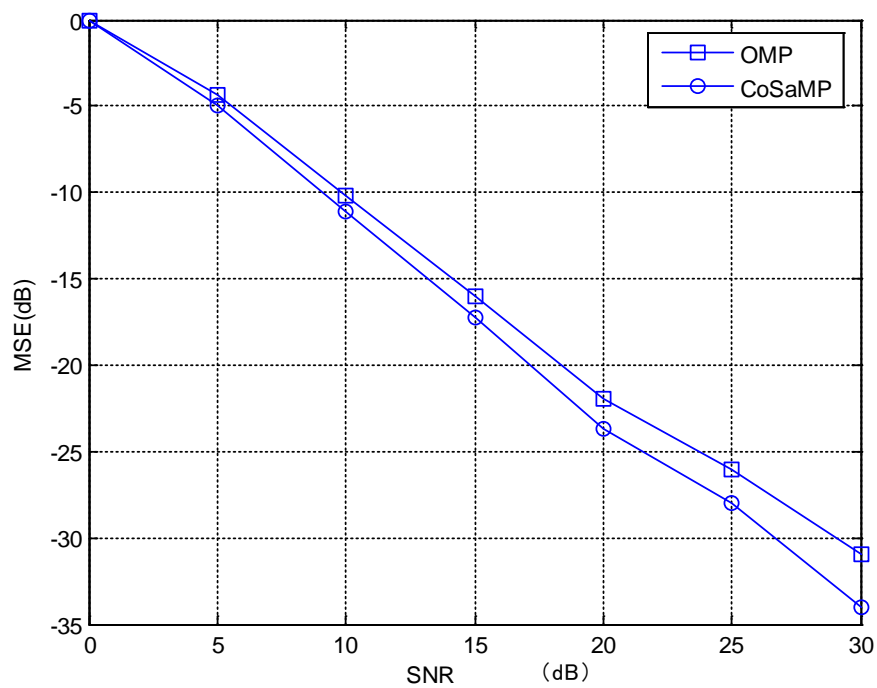

Figure 3. MSE of two algorithms

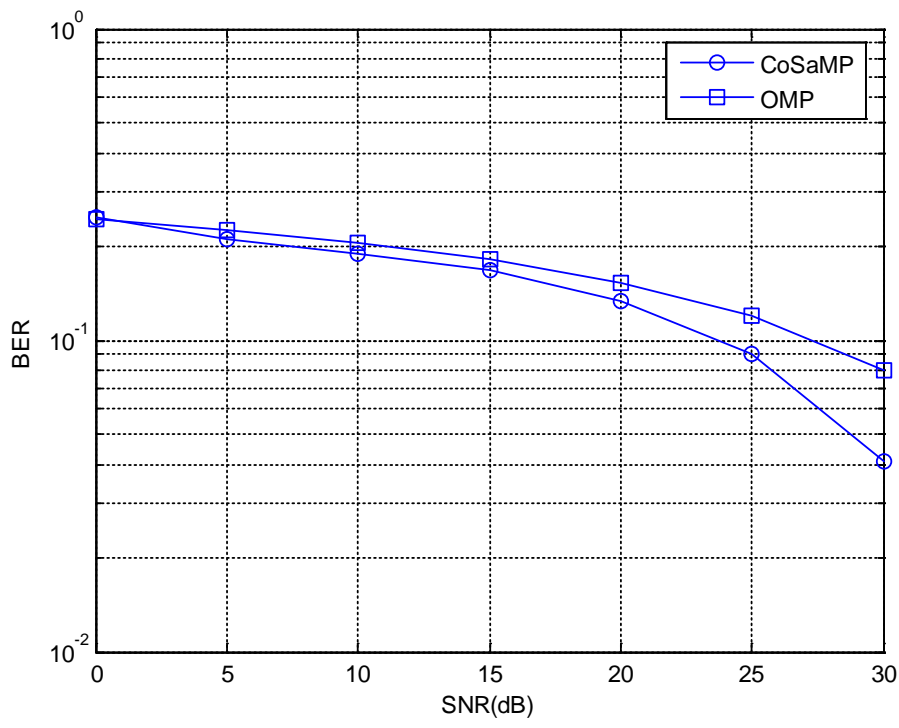

Figure 4. Comparison of two algorithms' BER when the number of pilots is 40 pilots 


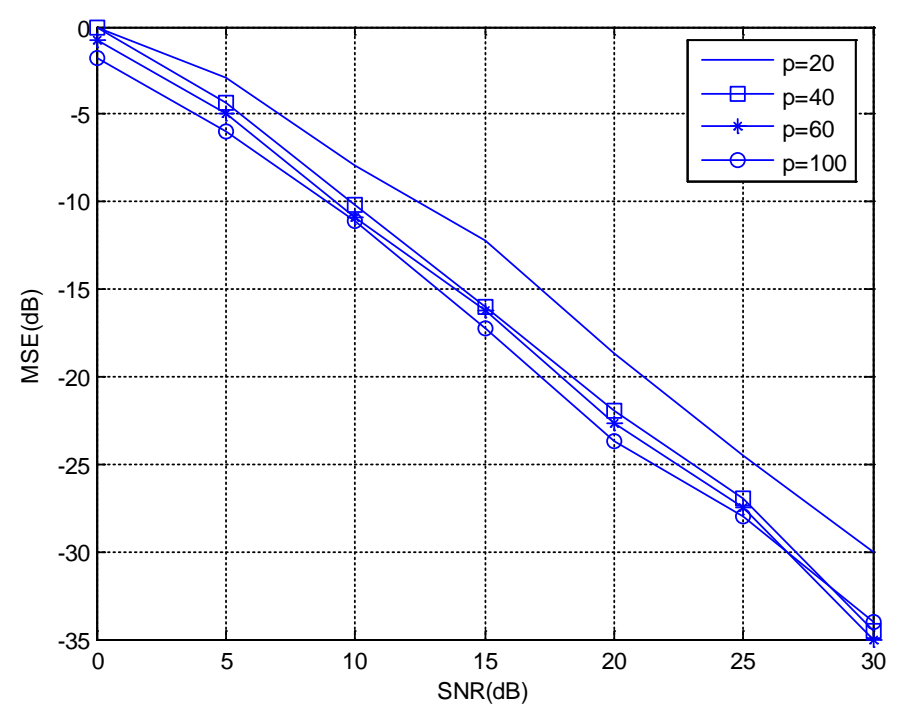

Figure 5. Comparison of CoSaMP`s BER when the number of pilots is different

For the performance comparison of OMP algorithm and CoSaMP algorithm, OFDM system with 200 sub-carriers, frequency band from $5 \mathrm{kHz}$ to $6 \mathrm{kHz}$ and multi-path channel are used for simulations. Additionally, the sampling frequency is $\mathrm{fs}=15 \mathrm{kHz}$, the symbol interval is $0.2 \mathrm{~s}$, and the guard time is $0.05 \mathrm{~s}$. The multi-path channel is shown in figure2.

Figure 3 is comparison of the OMP algorithm's and the CoSaMP algorithm's mean square error (MSE) when the pilot interval is 10 and the number of pilots is 20. From the figure 2 we can see that the MSE of CoSaMP algorithm is larger than the MSE of OMP algorithm. And when the SNR reaches to $30 \mathrm{~dB}$, two algorithm's MSE is below to 10-4.

As figure 4 shown that when the pilot interval is 5 and the number of pilots is 40 , the BERs of the UWA channel estimtion using two algorihms is low. In low SNR, the BERs of the UWA channel estimtion using two algorihms are little different. And in high, SNR the BER of the UWA channel estimtion using two algorihms is very different, here the BER of the UWA channel estimtion using OMP algorihm is higher than that of the UWA channel estimtion using CoSaMP algorihm . In 30dB the both are lower than $10 \%$.

Figure 5 is BER of CoSaMP algorithm used in UWA channel estimation when the number of pilots is 40,60 and 100 respectively. From that figure we can see that in low SNR more the pilots is adopted, the smaller MSE of that algorithm is obtained. This is because the estimation accuracy of CoSaMP algorithm depends largely on the number of pilots used in that algorithm. And the more pilots are used ,the more accurate the performance of that algorithm is. Moreover MSE curve with different pilot number is close, when the number of pilots is greater than 40 and SNR is high. And if more pilots are adopted, MSE could not be reduced, and the performance of the system could not be improved.

The above simulation results show that when using the same number of pilots, MSE of CoSaMP algorithm is lower than that of OMP algorithm, which makes the system BER using CoSaMP algorithm lower than that adopting OMP algorithm. And the difference of the both algorithms' MSE and BER are big. At the same time, notably when the number of pilots increasing to a certain value, the difference of MSE using different number of pilots is little. Therefore the optimum number of pilots adopted in CoSaMP algorithm is 40, when the number of subcarriers is 200.

\section{Conclusion}

In this paper two kinds of channel estimation algorithm, OMP and CoSaMP, based on compressed sensing are studied. Those algorithms are used and simulated in OFDM UWA communication system with the sparsity of the characteristics of UWA channel. The simulation results show that the estimation accuracy of CoSaMP algorithm with the global optimality is higher than that of OMP 
algorithm. At the same time, the optimum number of pilots adopted in CoSaMP algorithm is found, which could guarantee the better estimation performance with smaller number of pilots, and increase the throughput of the system effectively.

\section{References}

[1] CANDES E. Compressive sampling[C]: Proceedings of the International Congress of Mathematicians. Madrid, Spain: 2006:1433-1452Y. Mishing, in: Diffusion Processes in Advanced Technological Materials, edtied by D. Gupta Noyes Publications/William Andrew Publising, Norwich, NY (2004), in press.

[2] R. Baraniuk: Compressive Sensing, IEEE Signal Process. Mag., vol. 24, no. 4, July 2007, pp. 118-21.

[3] E. J. Candès and M. B. Wakin: An Introduction to Compressive Sampling, IEEE Signal Process. Mag., vol.25, no. 2, Mar. 2008, pp. 21-30.

[4] R. Chartrand et al.: Introduction to the Issue on Compressive Sensing, IEEE J. Selected Topics Signal Process., vol. 4, no. 2, Feb. 2010, pp. 241-43.

[5] B. Li, S. Zhou, M. Stojanovic, L. Freitag, and P. Willett: Multicarrier communication over underwater acoustic channels with nonuniform Doppler shifts, IEEE Journal of Oceanic Engineering, vol. 33, no. 2,pp. 198-209, Apr. 2008.

[6] C. R. Berger et al.: Sparse Channel Estimation for Multicarrier Underwater Acoustic Communication: From Subspace Methods to Compressed Sensing, IEEE Trans. Signal Process., vol.58, no. 3, Mar. 2010, pp. 1708-21.

[7] M. Stojanovic and J. Preisig: Underwater Acoustic Communication Channels: Propagation Models and Statistical Characterization, IEEE Commun. Mag., vol. 47, no. 1, Jan. 2009, pp. 84-89.

[8] W. G. Jeon, K. H. Paik, and Y. S. Cho: Efficient Channel Estimation Technique for OFDM Systems with Transmitter Diversity, Proc. IEEE Int’l. Symp. Personal, Indoor and Mobile Radio Commun., 2000,9(2):1246-1250)

[9] W. U. Bajwa et al.: Compressed Channel Sensing: A New Approach to Estimating Sparse Multipath Channels, Proc. IEEE, vol. 98, no. 6, June 2010, pp.1058-76

[10] D. Needell and J. A. Tropp: CoSaMP: Iterative Signal Recovery from Incomplete and Inaccurate Samples, Applied Comp. Harmonic Analysis, vol. 26, 2009, pp. 301-21. 